\title{
Effects of Weight Loss on Pericardial Fat and Left Ventricular Mass Assessed with Cardiac Magnetic Resonance Imaging in Morbid Obesity
}

\author{
Stephan M. Schneiter ${ }^{1}$, Ranjana Warrier ${ }^{2}$, Lisa Lefkovits ${ }^{1}$, Cheryl Laurie ${ }^{2}$, Paul E. O’Brien², \\ Andrew J. Taylor ${ }^{*}$ \\ ${ }^{1}$ Alfred Heart Centre and Baker IDI Heart and Diabetes Institute, Alfred Hospital, Melbourne, Australia; ${ }^{2}$ Centre for Obesity Re- \\ search and Education, Monash University Medical School, Alfred Hospital, Melbourne, Australia. \\ Email: *andrew.taylor@bakeridi.edu.au
}

Received March 29 ${ }^{\text {th }}$, 2011; revised May $16^{\text {th }}$, 2011; accepted July 20 ${ }^{\text {th }}, 2011$.

\begin{abstract}
Background: Obesity is a risk factor for cardiovascular disease and mortality. Significant weight loss has beneficial effects on left ventricular structure, in particular on regression of left ventricular hypertrophy $(L V H)$. We therefore evaluated the consequences of significant weight loss on left ventricular morphology, hemodynamics and pericardial fat. Methods: We performed volumetric cardiac magnetic resonance (CMR) imaging before and after significant weight loss due to laparoscopic adjustable gastric banding (LAGB). CMR was used to measure cardiac mass, volume and function, as well as to quantify pericardial fat. Results: Eleven patients (age $40.5 \pm 10$ yrs, body mass index $42.5 \pm 3.9$ $\mathrm{kg} / \mathrm{m}^{2}$ ) underwent CMR imaging before and a median 15.4 months after gastric banding. The BMI declined by $9.3 \pm 3.3$ $\mathrm{kg} / \mathrm{m}^{2}(p<0.001)$ with an average excess weight loss $(E W L)$ of $54.7 \% \pm 23.6 \%$. The left ventricular mass $(L V M)$ decreased from $130.0 \mathrm{~g} \pm 35.4 \mathrm{~g}$ to $117.0 \mathrm{~g} \pm 34.9 \mathrm{~g}(p=0.003)$, whereas the left ventricular chamber volumes and systolic function remained unchanged. After LAGB surgery the pericardial fat volume was reduced by $34.4 \mathrm{ml} \pm 22.1 \mathrm{ml}(p$ $<0.001)$. There was a significant correlation for change in pericardial fat volume with change in $L V M(R=0.67, p=$ $0.024)$, with no relationship between percentage of EWL and change in LVM $(p=0.49)$. Discussion: Significant weight loss in obesity is accompanied by a marked regression of $L V H$, with no apparent change in cardiac volume or function. The local effect of a diminished amount of pericardiac fat tissue may be more important than absolute weight loss with respect to the regression of $L V H$ in obesity.
\end{abstract}

Keywords: Cardiac Magnetic Resonance Imaging, Morbid Obesity, Left Ventricular Mass, Pericardial Fat

\section{Introduction}

Obesity is a global pandemic with significant implications for public health with respect to chronic illness including diseases of the cardiovascular system. Obesity increases the risk of adverse cardiac events indirectly by mediating risk factors associated with metabolic syndrome, thus propagating atherosclerosis with subsequent coronary artery disease. [1] However there are many direct effects of obesity on the heart and the cardiovascular system which can lead to increased morbidity and mortality. Obesity is associated with hemodynamic overload resulting in hyperdynamic circulation with a high stroke volume in order to meet the metabolic demands of the adipose tissue. [2,3] Several authors have demonstrated that obesity has resulted in increased left ventricular mass (LVM). [4-6] As a consequence cardiomyopathy of obese individuals often develops initially with diastolic dysfunction and possibly later in the course with worsening left ventricular systolic function. [7] A strong association between obesity and heart failure has also been described by the Framingham Heart Study. [8] Moreover obesity has been shown to be associated with cardiac arrhythmias such as bradyarrhythmias due to fatty replacement of the conduction system [9] as well as tachyarrhythmias with sudden cardiac death presumably due to prolonged QT interval [10].

Whilst the impact of obesity on the cardiovascular system is clearly apparent, there is also evidence that weight loss can be beneficial to the heart. However there are conflicting data in the literature relating to prognosis, 
with some suggestion that cardiovascular disease and heart failure when present in obese subjects is associated with a more favourable outcome [11], a phenomenon that has been labeled as the "obesity paradox". Therefore understanding the ways in which obesity modulates prognosis is of crucial importance. Visceral obesity plays a major role in the development of an unfavorable cardiovascular risk profile and is associated with increased cardiovascular morbidity and mortality. [12-15] Pericardial fat as an ectopic fat depot correlates with body mass index, visceral abdominal tissue, metabolic risk factors and coronary artery disease. [16-19] There is now evidence suggesting that the metabolic highly active epicardial adipose tissue (surrounding the coronary arteries) has a local, paracrine effect on the development of atherosclerosis in the epicardial coronaries with a significant association of epicardial/ pericardial fat volume with the prevalence of cardiovascular disease and myocardial infarction [19,20].

Cardiac magnetic resonance imaging (CMR) yields accurate and highly reproducible assessment of left ventricular mass. [21] It has been shown that the assessment of the amount of epicardial fat assessed by a CMR-based volumetric approach is superior to conventional imaging techniques such as echocardiography [22] and accurately reflects pericardial adipose tissue mass [23].

We therefore utilized CMR to evaluate the beneficial effect on the heart with respect to reduction of left ventricular parameters and pericardial fat following weight loss in otherwise healthy morbidly obese patients who underwent bariatric surgery. Weight loss was achieved with bariatric surgery using laparoscopic adjustable gastric banding (LAGB). This provides a safe and reliable method of achieving and sustaining significant weight loss in the majority of obese subjects. [24,25] It consists of placing an adjustable silicone gastric band around the cardia of the stomach. The band is adjustable by injection of saline into a peripherally placed reservoir. A correctly adjusted band provides early and prolonged satiety following a small meal, thereby reducing energy intake [26].

\section{Methods}

\subsection{Study Population and Assessment}

In this study 11 healthy obese subjects were included with the following inclusion criteria: age between 20 and 50 years, a current body mass index greater than 40 $\mathrm{kg} / \mathrm{m}^{2}$, absolute weight less than $150 \mathrm{~kg}$, normal electrocardiogram and no clinical evidence of cardiomyopathy. Patients were excluded from participation if they suffered from any condition that precluded them from CMR (such as non-compatible metallic implant and claustrophobia). Subjects were recruited presenting for LAGB at an ac- credited bariatric clinic in Melbourne and found to fulfill the basic entry criteria. The study was approved by the local ethics committee and informed written consent was obtained from each patient prior to enrolment.

\subsection{Clinical Evaluation}

Clinical assessment included physical examination and collection of weight and other parameters, including hip and waist diameter before intervention. Body mass index (BMI) was calculated as weight in kilograms divided by height in meters squared. Excess weight was defined as kilograms of weight above a BMI of 25 . The percentage of excess weight loss (EWL) was amount of weight loss divided by excess weight multiplied by 100 .

\subsection{CMR Protocol}

CMR was performed pre- and 1 year post-bariatric surgery on a clinical 1.5T-scanner (Signa HD 1.5T, GE Healthcare, Waukesha, Wisconsin, USA) using a dedicated 8-element phased-array cardiac coil. All sequences were acquired with ECG-triggering during a breath-hold. Left ventricular function was assessed by a steady state free precession pulse sequence (repetition time [TR] 3.1 ms, echo time [TE] $1.4 \mathrm{~ms}$, flip angle $45^{\circ}, 30$ phases, slice thickness $8 \mathrm{~mm}$, matrix $224 \times 224$, FOV $360 \mathrm{~mm}$ ). Standard long axis views and a short axis stack covering the whole left and right ventricle were obtained. For more distinct tissue differentiation, for example differentiation of pericardial fluid from fat seen on cine imaging, additional short axis dark blood prepared T1- and T2weighted images with a multislice turbo spin-echo sequences were obtained. Due to time constraints, short-axis T1- or T2-weigthed stacks were not acquired.

\subsection{Quantification of Cardiac Volume, Function and Pericardial Fat}

The short axis stack was analyzed using the summation of disc method by manually tracing the endocardial borders from the base to the apex at end-diastole and endsystole. The epicardial border was traced at end-diastole to yield myocardial mass. LVM (g) was calculated as the epicardial volume minus the endocardial volume multiplied by $1.05 \mathrm{~g} / \mathrm{cm}^{3}$ (specific gravity of myocardium). Cardiac output $(\mathrm{l} / \mathrm{min})$ was calculated as the left ventricular stroke volume (ml) multiplied by the heart rate (bpm). Areas of pericardial adipose tissue were assessed by tracing the end-diastolic short-axis images beginning at the most basal slice at the level of the mitral valve proceeding through the short-axis stack to the most apical slice. To assess epicardial fat volume, two lines were drawn: the first line was drawn along the outer myocardial border; the second line was drawn at the visceral 
pericardial layer, subtending an area of epicardial fat between the lines. Pericardial fat volume was assessed by including all the fat surrounding the heart (Figure 1). Epicardial and pericardial fat areas were calculated and volumes derived using the summation of disc method. Total epi- and pericardial fat mass were obtained by multiplying epicardial and pericardial fat volumes by the specific weight of fat $\left(0.92 \mathrm{~g} / \mathrm{cm}^{3}\right)$ [27].

\subsection{Statistical Analysis}

Demographic and anthropometric data are presented as mean values \pm standard error (SE). The Student $t$ test for paired collectives was used to compare pre- and post-surgical epi- and pericardial fat volumes, left ventricular (LV) volumes and LVM. To determine the relationship between linear variables the Pearson's correlation coefficient was calculated. A two-tailed p-value of $<0.05$ was considered significant for all comparisons.

\section{Results}

\subsection{Patient Demographics}

11 morbidly obese patients were studied. Their characteristics are shown in Table 1. Their mean age was 40.5 \pm 3.1 and $72.7 \%$ were of female gender $(\mathrm{n}=8)$, all patients were of Caucasian ancestry. All subjects underwent successful LAGB. Repeat follow up visits including CMR were obtained $15.4 \pm 1.3$ months after surgery.

\subsection{Clinical Effects of Bariatric Surgery}

Anthropometric parameters and blood pressures with the changes after surgery are listed in Table $\mathbf{1}$. There was a

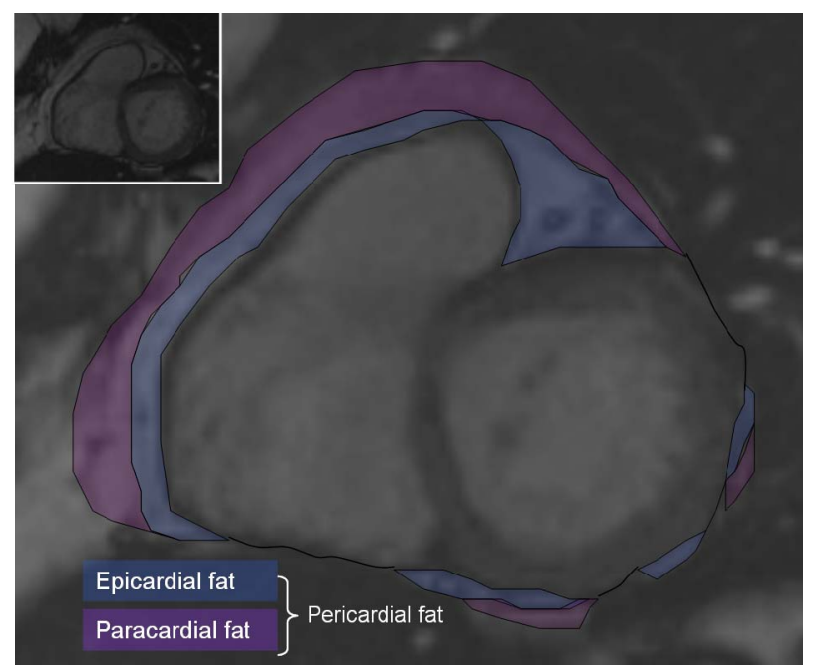

Figure 1. Pericardial adipose tissue. Basal short axis in end diastole: tracing the myocardial border, the pericardial border and the outer paracardial margin, both together representing the pericardial fat area, convertible to volumes.
Table 1. Anthropometric measures.

\begin{tabular}{cccc}
\hline Variable & Before surgery & After surgery & p-value \\
\hline LVEDV (ml) & $162.1 \pm 11.01$ & $165.5 \pm 10.5$ & 0.43 \\
LVESV (ml) & $68.4 \pm 4.1$ & $68.4 \pm 2.6$ & 0.98 \\
LVEF (\%) & $57.3 \pm 1.1$ & $57.9 \pm 1.6$ & 0.76 \\
Stroke volume (ml) & $93.7 \pm 7.4$ & $94.1 \pm 9.4$ & 0.93 \\
Heart rate (bpm) & $73.2 \pm 3.4$ & $60.2 \pm 2.6$ & 0.004 \\
Blood pressure & $80.0 \pm 3.7$ & $72.7 \pm 2.4$ & 0.14 \\
Diastolic (mmHg) & & & \\
\hline
\end{tabular}

highly significant reduction of weight by $26.0 \mathrm{~kg} \pm 2.5$ $\mathrm{kg}$ and body mass index which was reduced by $9.3 \mathrm{~kg} / \mathrm{m}^{2}$ $\pm 1.0 \mathrm{~kg} / \mathrm{m}^{2}$ with an average excess weight loss of $54.7 \%$ $\pm 7.1 \%$ after LAGB. No significant change of systolic or diastolic blood pressure was noted between baseline and follow up.

\subsection{Effects of Weight Loss on Cardiac Structure}

All baseline and follow up CMR were considered satisfactory for all measurements. Changes in cardiac structure and function with weight loss are listed in Table 2. The LVM decreased from $130.0 \mathrm{~g} \pm 10.7 \mathrm{~g}$ at baseline to $117.0 \mathrm{~g} \pm 10.5 \mathrm{~g}$ after weight loss $(\mathrm{p}=0.003)$ whereas the LV enddiastolic and endsystolic chamber volumes remained unchanged. The heart rate dropped significantly by $17.8 \%$.

\subsection{CMR Assessment of Epicardial and Pericardial Fat}

Table 2 shows pre- and post-surgical epi- and pericardial fat volume/mass. After gastric banding the overall epicardial fat volume was significantly reduced by $30.4 \mathrm{ml}$ $\pm 10.2 \mathrm{ml}$ and the pericardial fat volume was reduced by $34.3 \mathrm{ml} \pm 6.7 \mathrm{ml}(\mathrm{p}<0.001)$.

To assess inter- and intraobserver reproducibility, a second independent blinded observer repeated peri- and epicardial fat measurements. Intraobserver variability for the assessment of epicardial fat was poor with a nonsignificant correlation coefficient of $0.58(p=0.06)$. Interobserver variability for the volumetric assessment of epicardial fat was reasonable with a correlation coefficient of $0.70(p<0.05)$, whereas the correlation of assessment of pericardial fat between two independent observers was excellent with a coefficient of 0.97 (p < $0.001)$.

\subsection{Correlation of Pericardial Fat with Clinical Measures and Ventricular Structure at Baseline}

Table 3 lists associations of 'baseline’ pericardial fat vol- 
Table 2. CMR measurements.

\begin{tabular}{cccc}
\hline Variable & Before surgery & After surgery & p-value \\
\hline LVEDV (ml) & $162.1 \pm 11.01$ & $165.5 \pm 10.5$ & 0.43 \\
LVESV (ml) & $68.4 \pm 4.1$ & $68.4 \pm 2.6$ & 0.98 \\
LVEF (\%) & $57.3 \pm 1.1$ & $57.9 \pm 1.6$ & 0.76 \\
Stroke volume (ml) & $93.7 \pm 7.4$ & $94.1 \pm 9.4$ & 0.93 \\
Heart rate (bpm) & $73.2 \pm 3.4$ & $60.2 \pm 2.6$ & 0.004 \\
Cardiac output (l/min) & $6.8 \pm 0.5$ & $5.7 \pm 0.4$ & 0.07 \\
$\begin{array}{c}\text { LV mass (g) } \\
\text { Pericardial fat volume } \\
\text { (ml) }\end{array}$ & $130.0 \pm 10.7$ & $117.0 \pm 10.5$ & 0.003 \\
$\begin{array}{c}\text { Pericardial fat mass (g) } \\
\text { Epicardial fat volume } \\
\text { (ml) }\end{array}$ & $148.7 \pm 18.2$ & $117.2 \pm 20.0$ & $<0.001$ \\
$\begin{array}{c}\text { Epicardial fat mass (g) } \\
\text { (m) }\end{array}$ & $76.7 \pm 9.8$ & $48.7 \pm 5.7$ & 0.01 \\
\hline
\end{tabular}

Table 3. Correlations with pericardial fat.

\begin{tabular}{ccc}
\hline Variable & r & p-value \\
\hline Waist to hip ratio & 0.62 & 0.04 \\
Body mass index & -0.07 & 0.8 \\
Age & -0.13 & 0.73 \\
Left ventricular mass & 0.77 & 0.005 \\
\hline
\end{tabular}

ume with 'baseline' anthropometric measurements, LVM and age. There was a significant linear correlation between pericardial fat and LVM (Figure 2) as well as waste to hip ratio. There was no correlation with BMI or age and ventricular geometry. Furthermore there was a significant correlation between the change in pericardial fat volume and the change in LVM $(r=0.67, p=0.024)$. There was no correlation between the percentage of EWL and the percentage of change in LVM $(r=0.23$, $p$ $=0.49$ ).

\section{Discussion}

This prospectively conducted study demonstrated a decrease in the total amount of peri- and epicardial fat assessed by using CMR imaging in morbidly obese patients after bariatric surgery. This was accompanied by a reduction in LVM and heart rate in the absence of any significant change in ventricular volume, systolic ejection fraction, stroke volume or cardiac output. A striking finding in our study was that in addition to the correlation between baseline pericardial fat and LVM, the reduction in pericardial fat also correlated with regression of LVM

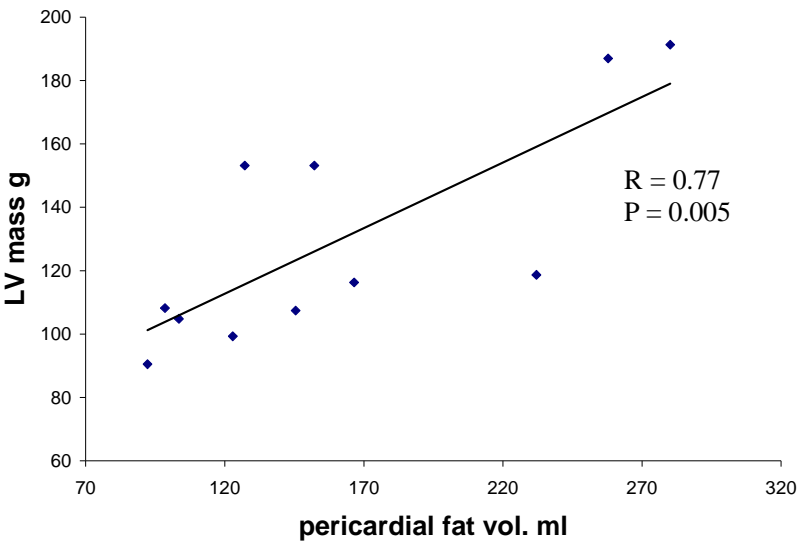

Figure 2. Correlation graph of pericardial fat volume and mass.

suggesting a mechanistic role of pericardial fat in the development of obesity-induced left ventricular hypertrophy (LVH). Unlike pericardial fat, there was no correlation between EWL and the change in LVM after surgery. Taken together these findings imply that reduction in pericardial fat, rather than absolute weight loss, may be the most important factor in regression of LVM in obesity. The amount of epicardial fat in obese patients is associated with body weight and also possibly with LVM as reported in autopsy studies [28,29]. Changes in pericardial fat mass could be shown after low calorie diet weight loss [30] and bariatric surgery assessed with echocardiography, [31] however echocardiographic measurements lack accuracy due to the reduced spatial resolution of echocardiography compared with CMR. Furthermore there are substantial individual differences in pericardial fat distribution, therefore single point measurements used in echocardiography are unlikely to accurately quantify the absolute amount of fat. It was first shown by Fluechter et al. that CMR measurement of epicardial fat volume is feasible, [22] and CMR has recently been validated in the assessment of pericardial adipose tissue volume in an ovine model showing a strong correlation between CMRderived paracardial adipose tissue volume and ex vivo paracardial fat mass [23].

The metabolically active epicardial fat, comparable to visceral fat deposits, is associated with cardiovascular disease, its risk factors and the metabolic syndrome [14,32] as well as with systemic markers of inflammation. [33] Paracrine and vasocrine signaling from the epicardial fat to the coronary arteries [34], given the short anatomical distance and the fact that they share the same microcirculation, is hypothesized to be a major contributor to coronary atherosclerosis. Therefore a reduced amount of epicardial and/or pericardial fat might lower the risk of coronary artery disease. It has recently been demonstrated 
that the volume of pericardial fat, measured by computed tomography, is independently associated with calcified coronary plaque [35] and it has also been shown in large population studies that pericardial fat and visceral adipose tissue are associated with cardiovascular disease independent of traditional measures of obesity. [19,20] Our data support the hypothesis that pericardial fat may play a similar paracrine role in the development of LVH in obesity.

There are however confusing specifications regarding measurements and discriminations of peri- and epicardial fat in the literature as was recently pointed out by Iacobellis. [36] The embryogenesis and the physiological role of the pericardial and epicardial fat are obviously very different and must be distinguished. The epicardial fat is located between the myocardium and the visceral layer of the pericardium, the pericardial fat is anterior to the epicardial fat and is located between the visceral and parietal pericardium, therefore also defined as any adipose tissue located within the pericardial sac. [37] Iacobellis and coworkers demonstrated in several publications the role of epicardial fat in predicting metabolic syndrome [38], insulin resistance [39] and subclinical atherosclerosis [40]. However, as it was not distinguished between the anatomical separate fat deposits of the heart, the above mentioned association has not clearly been shown to date for the pericardial fat. Our measurements revealed a poor intra- and interobserver variability to accurately assess the epicardial fat volume. This is mainly due to the difficulty in identifying the visceral pericardial layer, which is only clearly visible around the right ventricle at the basal level in a short axis cine imaging stack with CMR.

When interpreting our data a number of limitations need to be considered. The small sample size in our study may have limited the ability to demonstrate other changes and correlations of smaller magnitude. For example, heart rate was reduced following weight loss however this was not accompanied by a significant reduction in resting cardiac output, which may be a consequence of type II error reflective of the small numbers in our study. We also did not evaluate diastolic dysfunction or myocardial energetics, and systolic function was normal in our study population, therefore we cannot discount an effect of weight loss on cardiac function were more sensitive methods of assessment to be used. In addition metabolic parameters were not evaluated in this study as we focused on changes in anthropometric and imaging parameters of adiposity during weight loss. Although we demonstrated an association between reductions in pericardial fat and left ventricular mass, further detailed study in a larger group of patients is required to confirm the interplay between these factors as well as its putative mechanisms.
We have demonstrated that significant weight loss is associated with reduction in epicardial and pericardial fat which is accompanied by a reduction in left ventricular mass. The correlation between reduction of LVM and reduction in pericardial fat, but not total fat loss, implies a local effect of pericardial fat in the development of LVH in obesity. It could be anticipated that changes in pericardial fat, rather than total fat loss, might have greater prognostic benefits both with respect to limiting the development and progression of atherosclerosis, as well reducing the known harmful effects of LV hypertrophy. Future prospective studies in larger patient cohorts are indicated to evaluate the mechanism and effects of pericardial fat reduction in obese patients as well as its impact on hard clinical endpoints.

\section{REFERENCES}

[1] S. Malik, N. D. Wong, S. S. Franklin, et al., "Impact of the Metabolic Syndrome on Mortality from Coronary Heart Disease, Cardiovascular Disease, and All Causes in United States Adults," Circulation, Vol. 110, No. 10, 2004, pp. 1245-1250.

[2] J. K. Alexander, “Obesity and the Heart," Heart Disorders \& Disease, Vol. 2, No. 4, 1993, pp. 317-321.

[3] M. A. Alpert, "Obesity Cardiomyopathy: Pathophysiology and Evolution of the Clinical Syndrome," American Journal of the Medical Sciences, Vol. 321, No. 4, 2001, pp. 225-236. doi:10.1097/00000441-200104000-00003

[4] P. Friberg, A. Allansdotter-Johnsson, A. Ambring, et al., "Increased Left Ventricular Mass in Obese Adolescents," European Heart Journal, Vol. 25, No. 11, 2004, pp. 987-992. doi:10.1016/j.ehj.2004.03.018

[5] P. G. Danias, N. A. Tritos, M. Stuber, K. V. Kissinger, C. J. Salton and W. J. Manning, "Cardiac Structure and Function in the Obese: A Cardiovascular Magnetic Resonance Imaging Study,” Journal of Cardiovascular Magnetic Resonance, Vol. 5, No. 3, 2003, pp. 431-438. doi:10.1081/JCMR-120022259

[6] O. J. Rider, J. M. Francis, M. K. Ali, et al., "Determinants of Left Ventricular Mass in Obesity; A Cardiovascular Magnetic Resonance Study,” Journal of Cardiovascular Magnetic Resonance, Vol. 11, No. 1, 2009, p. 9. doi:10.1186/1532-429X-11-9

[7] I. De Scheerder, C. Cuvelier, R. Verhaaren, M. De Buyzere, G. De Backer and D. Clement, "Restrictive Cardiomyopathy Caused by Adipositas Cordis," European Heart Journal, Vol. 8, No. 6, 1987, pp. 661-663.

[8] S. Kenchaiah, J. C. Evans, D. Levy, et al., "Obesity and the Risk of Heart Failure," The New England Journal of Medicine, Vol. 347, 2002, pp. 305-313. doi:10.1056/NEJMoa020245

[9] A. M. Balsaver, A. R. Morales and F. W. Whitehouse, "Fat Infiltration of Myocardium as a Cause of Cardiac Conduction Defect,” American Journal of Cardiology, 
Vol. 19, No. 2, 1967, pp. 261-265.

doi:10.1016/0002-9149(67)90543-7

[10] W. B. Kannel, J. F. Plehn and L. A. Cupples, "Cardiac Failure and Sudden Death in the Framingham Study," American Heart Journal, Vol. 115, No. 4, 1988, pp. 869-875. doi:10.1016/0002-8703(88)90891-5

[11] C. J. Lavie, A. F. Osman, R. V. Milani and M. R. Mehra, "Body Composition and Prognosis in Chronic Systolic Heart Failure: The Obesity Paradox," American Journal of Cardiology, Vol. 91, No. 7, 2003, pp. 891-894. doi:10.1016/S0002-9149(03)00031-6

[12] S. M. Grundy, H. B. Brewer Jr., J. I. Cleeman, S. C. Smith Jr. and C. Lenfant, "Definition of Metabolic Syndrome: Report of the National Heart, Lung, and Blood Institute/American Heart Association conference on Scientific Issues Related to Definition," Circulation, Vol. 109, No. 3, 2004, pp. 433-438.

[13] K. M. Rexrode, V. J. Carey, C. H. Hennekens, et al., "Abdominal Adiposity and Coronary Heart Disease in Women," Journal of the American Medical Association, Vol. 280, No. 21, 1998, pp. 1843-1848.

[14] C. S. Fox, J. M. Massaro, U. Hoffmann, et al., "Abdominal Visceral and Subcutaneous Adipose Tissue Compartments: Association with Metabolic Risk Factors in the Framingham Heart Study,” Circulation, 2007, Vol. 116, No. 1, pp. 39-48.

[15] S. Yusuf, S. Hawken, S. Ounpuu, et al., "Obesity and the Risk of Myocardial Infarction in 27,000 Participants from 52 Countries: A Case-Control Study,” Lancet, Vol. 366, No. 9497, 2005, pp. 1640-1649.

[16] G. Iacobellis, M. C. Ribaudo, F. Assael, et al., "Echocardiographic Epicardial Adipose Tissue is Related to Anthropometric and Clinical Parameters of Metabolic Syndrome: A New Indicator of Cardiovascular Risk," The Journal of Clinical Endocrinology \& Metabolism, Vol. 88, No. 11, 2003, pp. 5163-5168. doi:10.1210/jc.2003-030698

[17] G. Iacobellis, F. Assael, M. C. Ribaudo, et al., "Epicardial Fat from Echocardiography: A New Method for Visceral Adipose Tissue Prediction," Obesity Research, Vol. 11, No. 2, 2003, pp. 304-310. doi:10.1038/oby.2003.45

[18] R. Taguchi, J. Takasu, Y. Itani, et al., "Pericardial Fat Accumulation in Men as a Risk Factor for Coronary Artery Disease,” Atherosclerosis, Vol. 157, No. 1, 2001, pp. 203-209.

[19] G. A. Rosito, J. M. Massaro, U. Hoffmann, et al., "Pericardial Fat, Visceral Abdominal Fat, Cardiovascular Disease Risk Factors, and Vascular Calcification in a Community-Based Sample: The Framingham Heart Study,” Circulation, Vol. 117, No. 5, 2008, pp. 605-613.

[20] A. A. Mahabadi, J. M. Massaro, G. A. Rosito, et al., "Association of Pericardial Fat, Intrathoracic Fat, and Visceral Abdominal Fat with Cardiovascular Disease Burden: The Framingham Heart Study,” European Heart Journal, 2009, Vol. 30, No. 7, pp. 850-856. doi:10.1093/eurheartj/ehn573
[21] S. G. Myerson, H. E. Montgomery, M. J. World and D. J. Pennell, "Left Ventricular Mass: Reliability of M-Mode and 2-Dimensional Echocardiographic Formulas," $\mathrm{Hy}$ pertension, Vol. 40, No. 5, 2002, pp. 673-678.

[22] S. Fluchter, D. Haghi, D. Dinter, et al., "Volumetric Assessment of Epicardial Adipose Tissue with Cardiovascular Magnetic Resonance Imaging," Obesity (Silver Spring), 2007, Vol. 15, No. 4, pp. 870-878.

[23] A. J. Nelson, M. I. Worthley, P. J. Psaltis, et al., "Validation of Cardiovascular Magnetic Resonance Assessment of Pericardial Adipose Tissue Volume,” Journal of Cardiovascular Magnetic Resonance, Vol. 11, 2009, p. 15. doi:10.1186/1532-429X-11-15

[24] P. E. O’Brien, J. B. Dixon, W. Brown, et al., "The Laparoscopic Adjustable Gastric Band (Lap-Band): A Prospective Study of Medium-Term Effects on Weight, Health and Quality of Life,” Obesity Surgery, Vol. 12, No. 5, 2002, pp. 652-660.

[25] A. E. Chapman, G. Kiroff, P. Game, et al., "Laparoscopic Adjustable Gastric Banding in the Treatment of Obesity: A Systematic Literature Review," Surgery, Vol. 135, No. 3, 2004, pp. 326-351.

[26] A. F. Dixon, J. B. Dixon, P. E. O’Brien, "Laparoscopic Adjustable Gastric Banding Induces Prolonged Satiety: A Randomized Blind Crossover Study," The Journal of Clinical Endocrinology \& Metabolism, Vol. 90, No. 2, 2005, pp. 813-819. doi:10.1210/jc.2004-1546

[27] W. S. Snyder, E. S. Nasset, L. R. Karhausen and G. P. Howells, "Report of the Task Group on Reference Man, International Comission on Radiological Protection," Pergamon Press, Oxford, 1975.

[28] J. Shirani, K. Berezowski and W. C. Roberts, "Quantitative Measurement of Normal and Excessive (Coradiposum) Subepicardial Adipose Tissue, Its Clinical Significance, and Its Effect on Electrocardiographic QRS Voltage," American Journal of Cardiology, Vol. 76, No. 5, 1995, pp. 414-418. doi:10.1016/S0002-9149(99)80116-7

[29] D. Corradi, R. Maestri, S. Callegari, et al., “The Ventricular Epicardial Fat Is Related to the Myocardial Mass in Normal, Ischemic and Hypertrophic Hearts," Cardiovascular Pathology, Vol. 13, No. 6, 2004, pp. 313-316.

[30] G. Iacobellis, N. Singh, S. Wharton and A. M. Sharma, "Substantial Changes in Epicardial Fat Thickness after Weight Loss in Severely Obese Subjects," Obesity (Silver Spring), Vol. 16, No. 7, 2008, pp. 1693-1697.

[31] H. J. Willens, P. Byers, J. A. Chirinos, E. Labrador, J. M. Hare and E. de Marchena, "Effects of Weight Loss after Bariatric Surgery on Epicardial Fat Measured Using Echocardiography,” The American Journal of Cardiology, Vol. 99, No. 9, 2007, pp. 1242-1245. doi:10.1016/j.amjcard.2006.12.042

[32] G. L. Vega, B. Adams-Huet, R. Peshock, D. Willett, B. Shah and S. M. Grundy, "Influence of Body Fat Content and Distribution on Variation in Metabolic Risk," The Journal of Clinical Endocrinology \& Metabolism, Vol. 91, No. 11, 2006, pp. 4459-4466. doi:10.1210/jc.2006-0814 
[33] K. M. Pou, J. M. Massaro, U. Hoffmann, et al., "Visceral and Subcutaneous Adipose Tissue Volumes Are CrossSectionally Related to Markers of Inflammation and Oxidative Stress: The Framingham Heart Study,” Circulation, Vol. 116, No. 11, 2007, pp. 1234-1241.

[34] H. S. Sacks and J. N. Fain, "Human Epicardial Adipose Tissue: A Review," American Heart Journal, Vol. 153, No. 6, 2007, pp. 907-917. doi:10.1016/j.ahj.2007.03.019

[35] J. Ding, S. B. Kritchevsky, T. B. Harris, et al., "The Association of Pericardial Fat with Calcified Coronary Plaque,” Obesity (Silver Spring), Vol. 16, No. 8, 2008, pp. 1914-1919. doi:10.1038/oby.2008.278

[36] G. Iacobellis, "Epicardial and Pericardial Fat: Close, but Very Different,” Obesity (Silver Spring), Vol. 17, No. 4, 2009, pp. 626-627.

[37] G. Iacobellis and H. J. Willens, "Echocardiographic Epicardial Fat: A Review of Research and Clinical Ap- plications," Journal of the American Society of Echocardiography, Vol. 22, No. 12, 2009, pp. 1311-1319. doi:10.1016/j.echo.2009.10.013

[38] G. Iacobellis, H. J. Willens, G. Barbaro, A. M. Sharma, "Threshold Values of High-Risk Echocardiographic Epicardial Fat Thickness,” Obesity (Silver Spring), Vol. 16, 2008, pp. 887-892.

[39] G. Iacobellis and F. Leonetti, "Epicardial Adipose Tissue and Insulin Resistance in Obese Subjects," The Journal of Clinical Endocrinology \& Metabolism, Vol. 90, No. 11, 2005, pp. 6300-6302. doi:10.1210/jc.2005-1087

[40] G. Iacobellis, A. M. Pellicelli, A. M. Sharma, B. Grisorio, G. Barbarini and G. Barbaro, "Relation of Subepicardial Adipose Tissue to Carotid Intima-Media Thickness in Patients with Human Immunodeficiency Virus,” American Journal of Cardiology, Vol. 99, No. 10, 2007, pp. 14701472. doi:10.1016/j.amjcard.2006.12.082 